\title{
Platelet Lyophilization and Cryopreservation: A Means of Generating a Standardized In-Vitro Human Blood Clot
}

\author{
Alexei Christodoulides ${ }^{1}$ and Nathan J. Alves, $\mathrm{PhD}^{1}$ \\ Indiana University School of Medicine: Department of Emergency Medicine ${ }^{1}$
}

\section{Background and Hypothesis:}

Numerous models, both in-vitro and in-vivo, exist for the analysis of blood-clotting pharmacodynamics. However, reliance on animal models, fresh blood, or lack of a complete component profile translates to little standardization/reproducibility of clotting parameters. Given the above limitations, the goal of our study was to generate a reproducible, physiologic human blood clot through cryopreservation and lyophilization of platelets. We hypothesized that the platelets exposed to either lyophilization or cryopreservation would have a prolonged ability to generate reproducible clots; however, clotting functionality might exhibit a setvalue decrease resulting from storage procedures.

\section{Experimental Design or Project Methods:}

Initial efforts focused on understanding the metabolic effects of storing platelets, plasma, and red blood cells (RBCs) at either $25^{\circ} \mathrm{C}$ or $4^{\circ} \mathrm{C}$ over a period of $25-$ days. Glucose consumption was utilized as a proxy for metabolic activity and assessed using a glucose-hexokinase assay. Secondly, RBCs, platelets, and plasma were stored with the aim of maximizing functionality for longer periods: $\mathrm{RBCs}-4^{\circ} \mathrm{C}+\mathrm{CPDA} 1$ solution and platelets - cryopreserved $\left(-80^{\circ} \mathrm{C}\right)$ or lyophilized. Functionality of stored blood components was assessed over a period of 1-week via Thromboelastographic (TEG) kinetic clotting readouts. Blood samples were provided by healthy volunteers $(n=6)$.

\section{Results:}

Utilization of glucose by RBCs and platelets was significantly increased during storage at $25^{\circ} \mathrm{C}$ versus $4^{\circ} \mathrm{C}$, with RBCs maintaining very consistent glucose consumption rates compared to platelets. Lastly, by 1-week of storage, wholeblood is able to maintain consistent clotting better than samples utilizing cryopreserved platelets.

\section{Conclusion and Potential Impact:}

Through our work, we were able to generate a reproducible synthetic human blood clot after 1-week of whole-blood storage as described by TEG parameters; a major stride in the ultimate goal of generating a model for pharmaceutical testing. Although not all parameters were preserved, we were able to maintain relative differences, ensuring consistent coagulability even with cryopreserved platelets. Further work will be conducted to understand the effects of greater storage durations. 